PROCEEDINGS OF THE

AMERICAN MATHEMATICAL SOCIETY

Volume 139, Number 6, June 2011, Pages 1983-1987

S 0002-9939(2010)10717-7

Article electronically published on November 10, 2010

\title{
REMARKS ON A CONJECTURE OF CHABAUTY
}

\author{
HATEM HAMROUNI
}

(Communicated by Alexander N. Dranishnikov)

\begin{abstract}
The Chabauty conjecture for connected nilpotent Lie groups has been proved by S. P. Wang. We show that one reasoning flaw has infiltrated the proof. We therefore give a new proof of the validity of Chabauty's conjecture in this setup. More generally, we shall prove that the Chabauty conjecture is true for rigid lattices and Zariski dense lattices of connected solvable Lie groups. In particular, the Chabauty conjecture holds for solvable Lie groups of $(R)$-type.
\end{abstract}

\section{INTRODUCTION}

Let $G$ be a connected solvable Lie group. We denote by $\mathscr{C}(G)$ the space of closed subgroups of $G$ equipped with the Chabauty topology; this is a compact metrizable space ([3], 2]). An open prebase of this topology is provided by the sets

$$
\mathcal{O}_{1}(K)=\{H \in \mathscr{C}(G): H \cap K=\emptyset\}, \quad \mathcal{O}_{2}(V)=\{H \in \mathscr{C}(G): H \cap V \neq \emptyset\},
$$

where $V$ and $K$ run respectively over all open and compact subsets of $G$. A discrete subgroup $\Gamma$ of $G$ is called a lattice of $G$ if $G / \Gamma$ has a finite invariant measure (or equivalently, $G / \Gamma$ is compact ([1] $)$ ). The set of all lattices of $G$ is denoted by $\mathscr{S}(G)$. Let $\operatorname{Aut}(G)$ be the group of all continuous automorphisms of $G$. Equipped with the compact-open topology, $\operatorname{Aut}(G)$ is a Lie group. Aut $(G)$ operates continuously on $\mathscr{S}(G)$ (endowed with the induced topology) with operation defined by $(\phi, \Gamma) \longmapsto$ $\phi(\Gamma)$. For $\Gamma \in \mathscr{S}(G)$, we denote by $\operatorname{Orb}(\Gamma)$ the orbit of $\Gamma$ and by $\operatorname{Stab}(\Gamma)$ the isotropy subgroup at $\Gamma$. In [5], C. Chabauty proposed the following conjecture.

Conjecture 1.1. For any lattice $\Gamma$ of $G, \operatorname{Orb}(\Gamma)$ with induced topology from $\mathscr{S}(G)$ is homeomorphic to $\operatorname{Aut}(G) / \operatorname{Stab}(\Gamma)$, or equivalently $\operatorname{Orb}(\Gamma)$ is locally compact.

The Chabauty conjecture is true in the case when $G=\mathbb{R}^{n}$. Moreover, for $\Gamma \in \mathscr{S}\left(\mathbb{R}^{n}\right)$ we have $([3]$, p. 187)

$$
\operatorname{Orb}(\Gamma)=\mathscr{S}\left(\mathbb{R}^{n}\right) \simeq \mathbf{G l}(n, \mathbb{R}) / \mathbf{G l}(n, \mathbb{Z}),
$$

where $\simeq$ denotes homeomorphism. In [14], S. P. Wang showed the validity of Chabauty's conjecture in the nilpotent case (see also [15]):

Theorem 1.2. The Chabauty conjecture is true for connected, simply connected nilpotent Lie groups.

Received by the editors May 27, 2010 and, in revised form, June 3, 2010.

2010 Mathematics Subject Classification. Primary 20E36, 20F16.

Key words and phrases. Chabauty topology, solvable Lie group, discrete uniform subgroup.

(C)2010 American Mathematical Society 
Furthermore, we have

Proposition 1.3 (14, Corollary 8.2). Let $G$ be a connected simply connected nilpotent Lie group. If $G$ has a lattice $\Gamma$, then $\mathscr{S}(G)$ is homeomorphic to $\operatorname{Aut}(G) /$ $\operatorname{Stab}(\Gamma)$.

The proof is based on the fact that $\mathscr{S}(G)$ is locally compact combined with the following result ([14, p. 525]).

Proposition 1.4. If $G$ is a connected simply connected nilpotent Lie group such that $\mathscr{S}(G) \neq \emptyset$, then the action $\operatorname{Aut}(G) \curvearrowright \mathscr{S}(G)$ is transitive.

The above proposition is incorrect. In this paper we shall give a counterexample to Proposition 1.4, on which Wang relies in proving Theorem 1.2 and Proposition 1.3. On the other hand, we contend that Proposition 1.3 is false. We provide a counterexample. For the rest of this paper we are concerned with studying Chabauty's conjecture for solvable Lie groups. It is well-known that the Chabauty conjecture is not true in this setup. A counterexample was given by S. P. Wang in 15. In sections 3 and 4 we prove that the Chabauty conjecture holds for every rigid lattice and every Zariski dense lattice of a connected simply connected solvable Lie group. In particular, we obtain the validity of Theorem 1.2

\section{Counterexamples to Proposition 1.3 and Proposition 1.4}

Consider the 3 -dimensional Heisenberg nilpotent Lie algebra $\mathfrak{h}_{3}=\mathbb{R}$-span $\{X, Y, Z\}$ with Lie brackets given by

$$
[X, Y]=Z
$$

and the non-defined brackets being equal to zero or obtained by antisymmetry. Let $H_{3}$ be the simply connected Lie group with Lie algebra $\mathfrak{h}_{3}$.

First, we give a counterexample to Proposition 1.4.

Proposition 2.1. Let $\mathrm{H}_{3}$ be the 3-dimensional Heisenberg group. The set

$$
\left\{\Gamma_{r}=\exp (\mathbb{Z} Z) \exp (\mathbb{Z} Y) \exp (r \mathbb{Z} X): r \in \mathbb{N}^{*}\right\}
$$

is a cross-section of $\operatorname{Aut}\left(\mathrm{H}_{3}\right)$-orbits in $\mathscr{S}\left(\mathrm{H}_{3}\right)$.

Proof. This follows from [1] (or [6]) and the Malcev rigidity theorem ([8]).

Remark 2.2. Recently, M. Bridson, P. De La Harpe and V. Kleptsyn obtained a more concrete description of orbits of $\operatorname{Aut}\left(H_{3}\right)$ in the space $\mathscr{S}\left(H_{3}\right)$ ([4, Section 7]).

Let us now give other counterexample to Proposition 1.4. A lattice $\Gamma$ of a connected, simply connected nilpotent Lie group $G$ is called $\log$-lattice if $\log (\Gamma)$ is an additive subgroup of $\mathfrak{g}([9])$. Let us denote by $\mathscr{R}(G)$ the set of log-lattices of $G$. From Theorem 2 of [9] it follows that $\mathscr{R}(G) \neq \emptyset$ if and only if $\mathscr{S}(G) \neq \emptyset$.

Proposition 2.3. Let $G$ be a connected simply connected nilpotent Lie group such that $\mathscr{R}(G) \varsubsetneqq \mathscr{S}(G)$. Then the action $\operatorname{Aut}(G) \curvearrowright \mathscr{S}(G)$ is not transitive.

Proof. Let $\Gamma \in \mathscr{R}(G)$ and $\phi \in \operatorname{Aut}(G)$. Let $\phi_{*} \in \operatorname{Aut}(\mathfrak{g})$ be the derivative of $\phi$ at identity. Since $\phi \circ \exp =\exp \circ \phi_{*}$, then $\phi(\Gamma) \in \mathscr{R}(G)$, and therefore $\operatorname{Orb}(\Gamma) \subset \mathscr{R}(G)$. Consequently, $\operatorname{Orb}(\Gamma) \neq \mathscr{S}(G)$.

Remark 2.4. The 3-dimensional Heisenberg group $H_{3}$ satisfies the above condition of Proposition 2.3. In fact, the lattice $\Gamma_{r}=\exp (\mathbb{Z} Z) \exp (\mathbb{Z} Y) \exp (r \mathbb{Z} X),\left(r \in \mathbb{N}^{*}\right)$ is a log-lattice if and only if $r$ is even ([9, p. 155]). 
We now present a counterexample to Proposition 1.3.

Proposition 2.5. Let $H_{3}$ be the 3-dimensional Heisenberg group. For every $\Gamma \in$ $\mathscr{S}\left(H_{3}\right), \mathscr{S}\left(H_{3}\right)$ is not homeomorphic to $\operatorname{Aut}\left(H_{3}\right) / \operatorname{Stab}(\Gamma)$.

We need the following lemma obtained by combining Proposition 5.3 and Proposition 6.3 of [4].

Lemma 2.6. For every $r \in \mathbb{N}^{*}$, the orbit $\operatorname{Orb}\left(\Gamma_{r}\right)$ of $\Gamma_{r}$ is connected and open in $\mathscr{S}\left(H_{3}\right)$.

Proof of Proposition 2.5. It follows from Lemma 2.6 that for every $r \in \mathbb{N}^{*}$ the orbit $\operatorname{Orb}\left(\Gamma_{r}\right)$ of $\Gamma_{r}$ is a connected component of $\mathscr{S}\left(H_{3}\right)$. Then, $\mathscr{S}\left(H_{3}\right)$ is not connected. On the other hand, the homogeneous space $\operatorname{Aut}\left(H_{3}\right) / \operatorname{Stab}(\Gamma)$ is connected (44). This completes the proof.

\section{The Chabauty COnjecture And Rigidity}

Definition 3.1. A lattice $\Gamma$ of $G$ is said to be rigid if for any isomorphism $\phi: \Gamma \longrightarrow$ $\Gamma^{\prime}$ of $\Gamma$ onto another lattice $\Gamma^{\prime}$ of $G$ there exists an automorphism $\widehat{\phi}: G \longrightarrow G$ such that $\left.\widehat{\phi}\right|_{\Gamma}=\phi$.

Theorem 3.2. The Chabauty conjecture is true for every rigid lattice of a connected, simply connected solvable Lie group.

Proof. Let $G$ be a connected solvable Lie group. Let $\mathscr{D}(G)$ be the set of all discrete subgroups of $G$. The space $\mathscr{D}(G)$ is open in $\mathscr{C}(G)$ ([2, Proposition E 1. 5]). On the other hand, if $\Gamma \in \mathscr{S}(G)$, then there is a neighbourhood of $\Gamma$ in $\mathscr{D}(G)$ consisting entirely of groups isomorphic to $\Gamma(7)$. Then, $\mathscr{S}(G)$ is open in $\mathscr{C}(G)$. Furthermore, the set $\mathscr{S}(G, \Gamma)$ of subgroups in $\mathscr{S}(G)$ which are isomorphic to $\Gamma$ is open and closed in $\mathscr{S}(G)$. Since $\mathscr{C}(G)$ is compact, $\mathscr{S}(G)$ and $\mathscr{S}(G, \Gamma)$ are locally compact. On the other hand, if $\Gamma$ is rigid, then $\mathscr{S}(G, \Gamma)=\operatorname{Orb}(\Gamma)$. It follows that $\operatorname{Orb}(\Gamma)$ is locally compact.

A connected, simply connected solvable Lie group $G$ is said to be of $(R)$-type if every eigenvalue $\lambda$ of any operator $\operatorname{Ad}(g), g \in G$, is purely real. Note that, obviously, any nilpotent Lie group is of $(R)$-type.

Theorem 3.3 ([12], [10]). All discrete uniform subgroups in a solvable Lie group of $(R)$-type are rigid.

Theorem 3.2 and Theorem 3.3 immediately imply the following result due to R. Mosak and M. Moskowitz [10] (see also [16]).

Corollary 3.4. The Chabauty conjecture is true in solvable Lie groups of $(R)$-type.

\section{Chabauty's conjecture And the Zariski Density}

Let $\mathcal{H}(\Gamma, G)$ be the topological space, with the topology of pointwise convergence, of all representations of $\Gamma$ into $G$. Let $\mathcal{R}(\Gamma, G)$ be the space of all isomorphisms $\phi: \Gamma \longrightarrow \phi(\Gamma)$ of $\Gamma$ onto another lattice of $G$. It is well known that $\mathcal{R}(\Gamma, G)$ is an open subset of $\mathcal{H}(\Gamma, G)$. Let $\mathcal{R}_{0}(\Gamma, G)$ be the connected component of the identity map $i_{\Gamma} \in \mathcal{R}(\Gamma, G)$. Any element $\phi \in \mathcal{R}_{0}(\Gamma, G)$ is said to be a deformation of $\Gamma$ in $G$. 
A subgroup $H$ of $G$ is called Zariski dense in $G$ if $H$ and $G$ have the same algebraic hull in the real algebraic group $\operatorname{Hol}(U)=\operatorname{Aut}(U) \cdot U$, where $U$ is the unipotent hull of $G([13])$.

Theorem 4.1 ([13], Corollary 7.3). Any Zariski dense lattice is rigid under deformations.

Theorem 4.2. The Chabauty conjecture holds for Zariski dense lattices of a connected, simply connected solvable Lie group.

Proof. Let $\Gamma$ be a Zariski dense lattice of a connected solvable Lie group $G$. We will prove that $\operatorname{Orb}(\Gamma)$ is closed in $\mathscr{S}(G)$. We recall that the space $\mathscr{S}(G)$ is metrizable ([2, Lemma E 1.1]). Let $\left(\Gamma_{n}\right)_{n \geq 0}$ be a sequence of points of $\operatorname{Orb}(\Gamma)$, which converges to $D$ in $\mathscr{S}(G)$. From [7, there exists a sequence $\left(\phi_{n}\right)_{n \geq 0}$ of $\mathcal{R}(D, G)$ converging to $i_{D}$ such that $\phi_{n}(D)=\Gamma_{n}$. On the other hand, there exists $m \in \mathbb{N}$ such that, for every $n \geq m, \phi_{n}$ belongs to a connected neighborhood of $i_{D}$ ([17]). It follows that, in particular, $\phi_{m} \in \mathcal{R}_{0}(D, G)$. By Theorem 4.1, let $\widehat{\phi}_{m} \in \operatorname{Aut}(G)$ be such that $\left.\widehat{\phi}_{m}\right|_{D}=\phi_{m}$. It follows that $D \in \operatorname{Orb}(\Gamma)$. Consequently $\operatorname{Orb}(\Gamma)$ is closed in $\mathscr{S}(G)$. This completes the proof.

\section{ACKNOWLEDGMENT}

It is a great pleasure to thank the anonymous referee for critical and valuable comments.

\section{REFERENCES}

[1] Auslander L., Green L., Hahn F., Flows on homogeneous spaces, Ann. of Math. Studies, no. 53, Princeton Univ. Press, 1963. MR167569 (29:4841)

[2] Benedetti R., Petronio C., Lectures on hyperbolic geometry, Springer, Berlin, 1992. MR.1219310 (94e:57015)

[3] Bourbaki N., Éléments de Mathématique, Intégration, Chapitres 7-8, Springer-Verlag, 2004. MR2098271 (2005f:28001)

[4] Bridson M., De La Harpe P., Kleptsyn V., The Chabauty space of closed subgroups of the three-dimensional Heisenberg group, Pacific J. Math., 240 (2009), 1-48. MR2485473 (2010a:22005)

[5] Chabauty C., Limite d'ensemble et géométrie des nombres, Bull. Soc. Math. France, 78 (1950), 143-151. MR0038983(12:479f)

[6] Gordon C. S., Wilson E. N., The spectrum of the Laplacian on Riemannian Heisenberg manifolds, Mich. Math. J., 33 (1986), 253-271. MR837583 (87k:58275)

[7] Macbeath A. M., Groups of homeomorphisms of a simply connected space, Ann. of Math. (2), 79 (1964), 473-488. MR0160848 (28:4058)

[8] Malcev A. I., On a class of homogeneous spaces, Amer. Math. Soc. Transl., 39 (1951), 276307. MR0039734 (12:589e)

[9] Moore C. C., Decomposition of unitary representations defined by discrete subgroups of nilpotent groups, Ann. of Math. (2), 82 (1965), 146-182. MR0181701(31:5928)

[10] Mosak R., Moskowitz M., Zariski density in Lie groups, Isr. J. Math., 52 (1985), 1-14. MR815596 (87h:22010)

[11] Raghunathan M. S., Discrete subgroups of Lie groups, Ergebnisse der Mathematik, Bd. 68, Springer, New York, 1972. MR0507234(58:22394a)

[12] Saitô M., Sur certain groupes de Lie résolubles II, Sci. Pap. Coll. Gen. Educ. Univ. Tokyo, 7 (1957), 157-168. MR0097463 (20:3932)

[13] Starkov A. N., Rigidity problem for lattices in solvable Lie groups, Pro. Indian Acad. Sci., 104 (1994), 495-514. MR.1314393 (96d:22017)

[14] Wang S. P., Limit of lattices in a Lie group, Trans. Amer. Math. Soc., 133 (1968), 519-526. MR0235066 (38:3378) 
[15] Wang S. P., On a conjecture of Chabauty, Proc. Amer. Math. Soc., 23 (1969), 569-572. MR0255728 (41:388)

[16] Wang S. P., Some properties of lattices in a Lie group, Illinois J. Math., 14 (1970), 35-39. MR0254176 (40:7386)

[17] Weil A., On discrete subgroups of Lie groups, Ann. of Math. (2), 72 (1960), 369-384. MR0137792 (25:1241)

Department of Mathematics, Faculty of Sciences at Sfax, University of Sfax, Route Soukra, B.P. 1171, 3000 Sfax, Tunisia

E-mail address: hatemhhamrouni@voila.fr 